\title{
Asymptotic distributions of estimated cyclic autocorrelations of DSSS signals and the applications
}

\author{
Dan $\mathrm{BaO}^{1 *}$, Riheng Wu${ }^{2}$, Jingjing $\mathrm{Cai}^{1}$ and Peng $\mathrm{Li}^{1}$
}

\begin{abstract}
Asymptotic distributions of estimated cyclic autocorrelations (CA) of direct sequence spread spectrum (DSSS) signals are derived in this paper. The estimation follows a zero-mean complex normal distribution in which the variance exhibits a cyclic thumbtack form, and the cyclic period equals the symbol period. This property of the estimated CA can be used in the detection and recognition problem of DSSS signals. The asymptotic performances of detection and recognition are carried out, and the simulations also verify the theoretical analysis.
\end{abstract}

\section{Introduction}

Direct sequence spread spectrum (DSSS) signals are widely used in commercial and military communications for their anti-jamming capabilities and low probability of interception. In DSSS systems, the information signal is modulated by a pseudo-noise $(\mathrm{PN})$ sequence before transmission. For cooperative communications, the PN spreading sequence is known to the receiver, which is used to carry out the despreading operation and recover the information data. Because of the processing gain in the matched filtering or correlation operations, the DSSS signals can be transmitted below the noise level. In the non-cooperative communication scenario, however, the receiver may have no priori knowledge of the transmitter's $\mathrm{PN}$ sequence. Hence, it is difficult for a non-cooperative receiver to detect and despread the DSSS signals because the PN sequence used by the transmitter is unknown to the receiver. Besides, one more important problem is the recognition of the DSSS signals, since one may usually want to know whether an intercepted signal is a DSSS signal or an ordinary pulse-amplitude modulated (PAM) signal in a non-cooperative condition. Also, the detection and recognition problems of DSSS signals have special significance for cognitive radio systems.

*Correspondence: dbao.2011@gmail.com

1 Department of Electronic Engineering, Xidian University, Xi'an 710071, China Full list of author information is available at the end of the article
To detect a DSSS communication hidden in the noise, a method that is based on the fluctuations of autocorrelation estimators is proposed by Burel in [1,2]. In [3], the detection, symbol period, and chip width estimation of DSSS signals are carried out based on delay-multiply, correlation, and spectrum analysis, respectively. In [4], Deng presents an autocorrelation estimation-based detection method. It is suitable for the real-time detection of DSSS signals at low signal-to-noise ratio (SNR) in a cognitive radio system. An approach is proposed in [5] to detect the baseband DSSS signal with narrowband interference based on blind source separation and fluctuations of the autocorrelation second moment. An algorithm for correlation-based detection of direct sequence spread spectrum signals with direction finding, including direction filtering and narrowband interference rejection, is implemented and evaluated in MATLAB in [6].

In [7], relying upon the asymptotic normality and consistency of $k$ th-order cyclic statistics, asymptotically optimal $\chi^{2}$ tests are developed to detect the presence of cycles in the $k$ th-order cyclic cumulants or polyspectra. The paper [8] deals with the analytical evaluation of the asymptotic detection and false alarm probabilities of multicycle and single-cycle detectors operating in additive white Gaussian noise, which are based on the cyclostationarity properties of the signal to be intercepted.

Self-recovering receivers for DSSS signals in multipath with unknown spreading codes are discussed in [9], wherein a zero-forcing receiver/equalizer is proposed to

\section{Springer}

(c) 2013 Bao et al. licensee Springer. This is an Open Access article distributed under the terms of the Creative Commons Attribution License (http://creativecommons.org/licenses/by/2.0), which permits unrestricted use, distribution, and reproduction in any medium, provided the original work is properly cited. 
recover the transmitted data. In [10], a method is proposed for estimating the pseudo-random sequence without any priori knowledge about the transmitter. Only the duration of the pseudo-random sequence is assumed to have been estimated. This approach is based on eigen analysis techniques.

Notice that the autocorrelation of DSSS signals is applied to resolve the detection problem in [1-6]. However, none of them deal with a theoretical interpretation for the fluctuation of the autocorrelation of DSSS signals and the asymptotic performances. Though the cyclic autocorrelation functions of the baseband DSSS PAM signal are derived in [11], the asymptotic distributions of the cyclic autocorrelations (CA) are absent. In this paper, we consider the asymptotic distributions of the estimated cyclic autocorrelations of DSSS signals, which may have the potential applications in non-cooperative communication environments. Then, the theoretical properties of the CA are applied in detection and recognition problems of DSSS signals. The remainder of the paper is organized as follows: In Section 2, we evaluate the distributions of the estimation of the cyclic autocorrelation, from which some interesting properties are found. In Section 2, we apply the result of the former section to the problem of DSSS detection and recognition, where the optimal threshold is given. Section 2 provides some simulation examples to illustrate the performance of the proposed algorithm. Finally, Section 2 concludes the paper.

\section{Asymptotic distributions of the estimation}

Consider a complex-valued continuous time series $x(t)$ with a zero mean. It exhibits wide-sense cyclostationarity if the cyclic autocorrelation function (CAF)

$$
R_{x x *}(\alpha ; \tau)=\lim _{T \rightarrow \infty} \frac{1}{T} \int_{-T / 2}^{T / 2} x(t+\tau) x^{*}(t) e^{-j 2 \pi \alpha t} d t
$$

is not identically zero when cycle frequency $\alpha \neq 0$, where * denotes conjugation.

A linear time-variant system with input-output relation [12]

$$
y(t)=\int_{R} h(t, u) x(u) d u,
$$

where $x(t)$ is the input, $y(t)$ is the corresponding output, and $h(t, u)$ is the impulse response function, is called a linear almost-periodically time-variant (LAPTV) system if the time-variant impulse response function can be expressed as the Fourier series expansion [12]

$$
h(t, u)=\sum_{\sigma \in G} h_{\sigma}(t-u) e^{j 2 \pi \sigma u},
$$

where $G$ is a countable set. The CAF of the output for the LAPTV system can be expressed as the convolution of the input CAF $R_{x x *}$ and the ambiguity function of $h(t, u)$ [12]

$$
R_{y y *}(\alpha ; \tau)=\sum_{\sigma_{1} \in G} \sum_{\sigma_{2} \in G}\left[R_{x x *}\left(\alpha-\sigma_{1}-\sigma_{2} ; \tau\right) e^{j 2 \pi \sigma_{1} \tau}\right] \underset{\tau}{\otimes} r_{12}(\alpha ; \tau),
$$

where $\underset{\tau}{\otimes}$ denotes convolution with respect to $\tau$, and $r_{12}(\alpha ; \tau)$ denotes the ambiguity function of $h(t, u)$

$$
r_{12}(\alpha ; \tau)=\int_{R} h_{\sigma_{1}}(\tau+s) h_{\sigma_{2}}(s) e^{-j 2 \pi \alpha s} d s .
$$

Equation 4 can be specialized to the case of the linear time-invariant systems for which the coefficient of the impulse response function is given by

$$
h(t+\tau, t)=h(\tau) .
$$

Hence, from (3) one has [13]

$$
h_{\sigma}(\tau)=h(\tau) \delta_{\sigma} \text {, }
$$

where $\delta_{\sigma}$ is the Kronecker delta, which $\delta_{\sigma}=1$ for $\sigma=0$ and $\delta_{\sigma}=0$ otherwise. It results in

$$
R_{y y *}(\alpha ; \tau)=R_{x x *}(\alpha ; \tau) \underset{\tau}{\otimes} r_{12}(\alpha ; \tau) .
$$

Consider a DSSS system, where the equivalent complex baseband received signal $r(t)$ contaminated by Gaussian noise can be expressed as

$$
r(t)=x(t)+n(t)
$$

where $x(t)$ is a DSSS PAM signal

$$
x(t)=A \sum_{k=-\infty}^{+\infty} a(k) p\left(t-k T_{b}\right) e^{j 2 \pi f_{c} t+\theta_{c}},
$$

where $A$ is a received amplitude, $f_{c}$ is a residual carrier, $\theta_{c}$ is an unknown carrier phase, and $n(t)$ is a complex additive white Gaussian noise with power spectrum density $N_{0}$.

In Equation 10, $\{a(k)\}_{k \in Z}$ is an information code sequence of independent and identically distributed binary symbols taking on values \pm 1 with equal probability, $T_{b}$ is the bit period, and

$$
p(t)=\sum_{n=0}^{N_{c}-1} c(n) q\left(t-n T_{c}\right)
$$

is a spreading waveform, where $\left\{c(n), n=0, \cdots, N_{c}-1\right\}$ is the $N_{c}$-length spread spectrum code sequence or called PN code sequence with $c(n) \in\{+1,-1\}, T_{c}$ is the chip period such that $T_{b}=N_{c} T_{c}$, and $q(t)$ is the chip pulse shape. Specifically, $q(t)$ is the rectangular pulse, in which $q(t)=\operatorname{rect}\left(t / T_{c}\right)$, where

$$
\operatorname{rect}(t)=\left\{\begin{array}{ll}
1 & |t| \leq 1 / 2 \\
0 & |t|>1 / 2
\end{array} .\right.
$$


For the convenience of analysis, Equation 10 can be rewritten as

$$
x(t)=A e^{j 2 \pi f_{c} t+\theta_{c}} x_{\delta}(t) \otimes p(t),
$$

where $\otimes$ denotes convolution, and $x_{\delta}(t)$ is the ideal sampled signal

$$
x_{\delta}(t)=\sum_{k=-\infty}^{+\infty} a(k) \delta\left(t-k T_{b}\right)
$$

with $\delta(t)$ denoting Dirac's delta. Now the CAF of $x(t)$ can be expressed as by using Equation 8

$$
R_{x x *}(\alpha ; \tau)=A^{2} e^{j 2 \pi f_{c} \tau} R_{x_{\delta} x_{\delta}^{*}}(\alpha ; \tau) \underset{\tau}{\otimes} r_{p p^{*}}(\alpha ; \tau),
$$

where $R_{x_{\delta} x_{\delta}^{*}}(\alpha ; \tau)$ is the CAF of $x_{\delta}(t)$,

$$
\begin{aligned}
R_{x_{\delta} x_{\delta}^{*}}(\alpha ; \tau)= & \lim _{T \rightarrow \infty} \frac{1}{T} \int_{-T / 2}^{+T / 2} \sum_{n=-\infty}^{+\infty} a(n) \delta\left(t+\tau-n T_{b}\right) \\
& \sum_{m=-\infty}^{+\infty} a(m) \delta\left(t-m T_{b}\right) e^{-j 2 \pi \alpha t} d t
\end{aligned}
$$

and $r_{p p^{*}}(\alpha ; \tau)$ is the ambiguity function (AF) of $p(t)$. The $\mathrm{AF} r_{p p^{*}}(\alpha ; \tau)$ in (15) can be expressed as [11]

$$
r_{p p^{*}}(\alpha ; \tau)=r_{q}(\alpha ; \tau) \underset{\tau}{\otimes} \gamma_{c}(\alpha ; \tau),
$$

where

$$
\begin{aligned}
r_{q}(\alpha ; \tau)= & \int_{-\infty}^{+\infty} q(t) q(t+\tau) e^{-j 2 \pi \alpha t} d t \\
= & \exp \left[-j 2 \pi \alpha\left(T_{c}-\tau\right)\right] \operatorname{rect}\left(\frac{\tau}{2 T_{c}}\right)\left(T_{c}-|\tau|\right) \\
& \times \operatorname{sinc}\left(\alpha\left(T_{c}-|\tau|\right)\right)
\end{aligned}
$$

and

$$
\gamma_{c}(\alpha ; \tau)=\sum_{n=0}^{N_{c}-1} \sum_{m=0}^{N_{c}-1} c(n) c(m) e^{-j 2 \pi \alpha T_{c} m} \delta\left(\tau-(n-m) T_{c}\right) .
$$

The AF is a useful tool to analyze the response of a matched receiver, which is often used to represent the magnitude of the matched receiver output for a coherent signal. Due to the white noise-like pseudo-noise sequence of the spreading waveform, the AF of $p(t)$ has a property of superb time-frequency concentration, which means that the energy of $p(t)$ is concentrated in a very small timefrequency region $-T_{c}<\tau<T_{c}$ and $-1 / T_{b}<\alpha<1 / T_{b}$. An example of the AF of the spreading waveform with $N_{c}=31$ and $T_{c}=1$ is plotted in Figure 1, which shows that the magnitude of $r_{p p^{*}}(\alpha ; \tau)$ looks like a thumbtack.

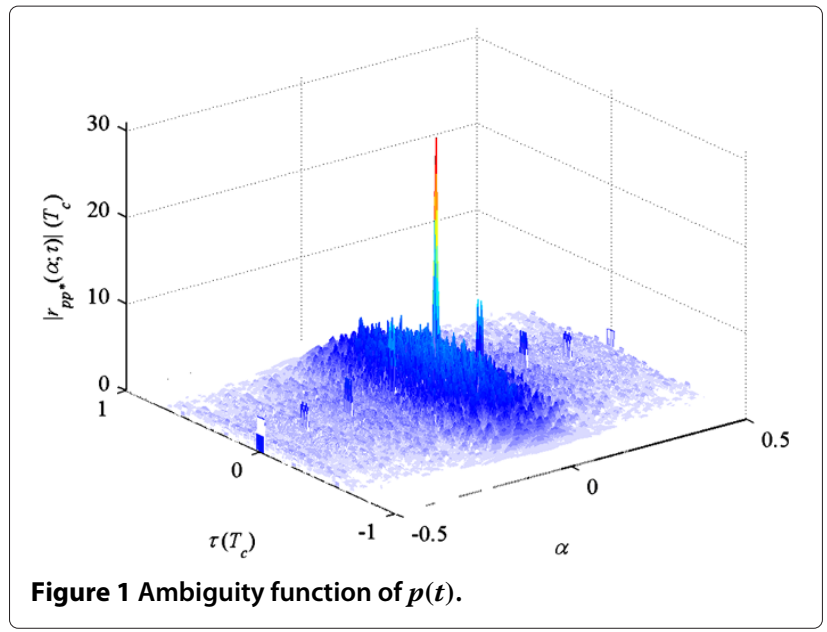

Take a closer look at the CAF of $x_{\delta}(t)$. Consider the following estimator of $R_{x_{\delta} x_{\delta}^{*}}(\alpha ; \tau)$ :

$$
\hat{R}_{x_{\delta} x_{\delta}^{*}}^{(T)}(\alpha ; \tau)=\frac{1}{T} \sum_{t=-T / 2}^{T / 2-1} x_{\delta}(t) x_{\delta}^{*}(t-\tau) e^{-j \alpha t} .
$$

For the whiteness of the sequence $\{a(k)\}_{k \in Z}$, one has the following expression for $\hat{R}_{x_{\delta} x_{\delta}^{*}}^{(T)}(\alpha ; \tau)$ :

$$
\hat{R}_{x_{\delta} x_{\delta}^{*}}^{(T)}(\alpha ; \tau)=\frac{1}{T_{b}} \delta_{\left(\alpha T_{b} \bmod 1\right.} \delta(\tau)+\sum_{n} \varepsilon^{(T)}(\alpha ; n) \delta\left(\tau-n T_{b}\right),
$$

where $n$ is an integer, $\delta_{n}$ is the Kronecker delta, $\delta(\cdot)$ denotes the Dirac delta function, mod means modulo operation, and $\varepsilon^{(T)}(\alpha ; n) \delta\left(\tau-n T_{b}\right)$ represents the estimation error which vanishes asymptotically as $T \rightarrow \infty$. The estimation error $\varepsilon^{(T)}(\alpha ; n) \delta\left(\tau-n T_{b}\right)$ was usually ignored in former literatures just as noises, while it has interesting uses in some special cases, such as in the DSSS systems, because it has non-zero values only when $\tau=n T_{b}$. It is due to the fact that one just considered the first non-zero term of the CAF (21) before, while the second vanishing term was rarely utilized. In our opinion, by properly making use of the vanishing term, we will show in the following part that the CAF exhibits some vanishing but useful properties in testing the presence of the DSSS signals.

Next, the asymptotical distribution of $\varepsilon^{(T)}(\alpha ; n)$ will be evaluated as follows. The estimator $\hat{R}_{x_{\delta} x_{\delta}^{*}}^{(T)}(\alpha ; \tau)$ defined in (20) is mean-square sense consistent [7], i.e.,

$$
\lim _{T \rightarrow \infty} \hat{R}_{x_{\delta} x_{\delta}^{*}}^{(T)}(\alpha ; \tau) \stackrel{\text { m.s.s. }}{=} R_{x_{\delta} x_{\delta}^{*}}(\alpha ; \tau) .
$$

Additionally, $\sqrt{T}\left[\hat{R}_{x_{\delta} x_{\delta}^{*}}^{(T)}(\alpha ; \tau)-R_{x_{\delta} x_{\delta}^{*}}(\alpha ; \tau)\right]$ is asymptotically complex normal [7]. From the asymptotic normality of CAF estimators that 


$$
\begin{aligned}
& \lim _{T \rightarrow \infty} \sqrt{T} \varepsilon^{(T)}(\alpha ; n) \delta\left(\tau-n T_{b}\right) \\
& \stackrel{D}{=} C N\left(0, \Gamma_{x_{\delta}}\left(\alpha ; n T_{b}\right), C_{x_{\delta}}\left(\alpha ; n T_{b}\right)\right), n \neq 0,
\end{aligned}
$$

where $\stackrel{D}{=}$ denotes convergence in distribution, $C N(\mu, \Gamma, C)$ stands for a complex normal density where $\mu$ is the location parameter, $\Gamma$ is the covariance matrix, and $C$ is the relation matrix [14]. From Theorem 1 in [7], the parameters in (23) can be given as

$$
\Gamma_{x_{\delta}}\left(\alpha ; n T_{b}\right)=S_{x_{\delta} x_{\delta} x_{\delta}^{*} x_{\delta}^{*}}^{0}(\alpha)_{n T_{b}, n T_{b}}
$$

and

$$
C_{x_{\delta}}\left(\alpha ; n T_{b}\right)=S_{x_{\delta} x_{\delta} x_{\delta} x_{\delta}}^{2 \alpha}(\alpha)_{n T_{b}, n T_{b}}
$$

where $S_{a b c d}^{\gamma}(\omega)_{\tau, \rho}^{(*)}$ are the (conjugated) cyclic cross spectrum which is defined as [7]

$$
\begin{aligned}
& S_{a b c d}^{\gamma}(\omega)_{\tau, \rho}=\lim _{T \rightarrow \infty} \frac{1}{T} \sum_{t=0}^{T-1} \sum_{\xi=-\infty}^{\infty} \\
& \operatorname{cum}(a(t) b(t+\tau), c(t+\xi) d(t+\xi+\rho)) e^{-j \omega \xi} e^{-j \gamma t} .
\end{aligned}
$$

The notation $\operatorname{cum}($.$) in (26) represents the cumulant$ which defined in [7]. As in [8], the cyclic spectrum of $x_{\delta}(t)$ can be written as

$$
S_{x_{\delta} x_{\delta}}^{\gamma}(\omega)=\frac{1}{T_{b}} \delta_{\left(\gamma T_{b}\right) \bmod 1}
$$

By substituting (14) into (26), using (27), the parameters of the cyclic cross spectrum can be simplified, accounting for the whiteness of the sequence $\{a(k)\}_{k \in Z}$ when $\tau=n T_{b}$ and $\tau \neq 0$

$$
\Gamma_{x_{\delta}}\left(\alpha ; n T_{b}\right)=\frac{1}{T_{b}}
$$

and

$$
C_{x_{\delta}}\left(\alpha ; n T_{b}\right)=\frac{1}{T_{b}} \delta_{\left(2 \alpha T_{b}\right) \bmod 1}
$$

From what that has been discussed above, we may draw a conclusion that if $\varepsilon^{(T)}(\alpha ; n) \delta\left(\tau-n T_{b}\right)$, the estimation error of $\hat{R}_{x_{\delta} x_{\delta}^{*}}^{(T)}(\alpha ; \tau)$ is not identically zero and behaves in a random manner when $\tau=n T_{b}$, and $\sqrt{T} \varepsilon^{(T)}(\alpha ; n)$ follows asymptotic normal distribution that

$$
\lim _{T \rightarrow \infty} \sqrt{T} \varepsilon^{(T)}(\alpha ; n) \stackrel{D}{=} C N\left[0, \frac{1}{T_{b}} \delta_{\left(2 \alpha T_{b}\right) \bmod 1}, \frac{1}{T_{b}}\right] .
$$

By substituting (21) into (15), the estimation of the CAF of $x(t)$ can finally be expressed as

$$
\begin{aligned}
\hat{R}_{x x *}(\alpha ; \tau)= & \frac{1}{T} \sum_{t=-T / 2}^{T / 2-1} x(t) x^{*}(t-\tau) e^{-j \alpha t} \\
= & A^{2} e^{j 2 \pi f_{c} \tau}\left\{\frac{1}{T_{b}} \delta_{\left(\alpha T_{b}\right) \bmod 1} r_{p p^{*}}(\alpha ; \tau)\right. \\
& \left.\left.+\sum_{n} \varepsilon^{(T)}(\alpha ; n) r_{p p^{*}}\left(\alpha ; \tau-n T_{b}\right)\right\}\right\},
\end{aligned}
$$

where $\sqrt{T} \varepsilon^{(T)}(\alpha ; n)$ is defined as in (30), and $r_{p p^{*}}(\alpha ; \tau)$ is defined as in (17). The first term in (31) is well discussed in [7], so we just focus on the second term. Though it vanishes asymptotically as $T \rightarrow \infty$, the second term does exist, and due to the thumbtack-like AF $r_{p p^{*}}(\alpha ; \tau)$ of $p(t)$, it makes some special peaks appear, in a stochastic manner, in the regions $n T_{b}-T_{c}<\tau<n T_{b}+T_{c}$ and $-1 / T_{b}<\alpha<1 / T_{b}$.

When $T \rightarrow \infty$, the CAF of $x(t)$ can be simplified to a theoretical expression as

$$
R_{x x *}(\alpha ; \tau)=\frac{A^{2} e^{j 2 \pi f_{c} \tau}}{T_{b}} \delta_{\left(\alpha T_{b}\right) \bmod 1} r_{p p^{*}}(\alpha ; \tau),
$$

which has also been discussed in [8].

From $(31), \hat{R}_{x x *}(\alpha ; \tau)$ follows an asymptotic complex normal distribution that

$$
\begin{aligned}
& \lim _{T \rightarrow \infty} \sqrt{T}\left[\hat{R}_{x x *}(\alpha ; \tau)-R_{x x *}(\alpha ; \tau)\right] \\
& \quad \stackrel{D}{=} C N\left[0, \Gamma_{x}(\alpha ; \tau), C_{x}(\alpha ; \tau)\right] .
\end{aligned}
$$

Since the support of $r_{p p^{*}}(\alpha ; \tau)$ is on $-N_{c} T_{c}<\tau<N_{c} T_{c}$ and $\varepsilon^{(T)}(\alpha ; n)$ is zero-mean complex normal distributed, the parameters in (33) can be defined as follows:

$$
\begin{aligned}
\Gamma_{x}(\alpha ; \tau)= & \frac{A^{4}}{T_{b}}\left[\left|r_{p p^{*}}\left(\alpha ; \tau \% 2 T_{b}\right)\right|^{2}+\left|r_{p p^{*}}\left(\alpha ;\left(\tau+T_{b}\right) \% 2 T_{b}\right)\right|^{2}\right] \\
C_{x}(\alpha ; \tau)= & \frac{A^{4}}{T_{b}} e^{j 4 \pi f_{c} \tau}\left[r_{p p^{*}}^{2}\left(\alpha ; \tau \% 2 T_{b}\right)+r_{p p^{*}}^{2}\left(\alpha ;\left(\tau+T_{b}\right) \% 2 T_{b}\right)\right] \\
& \delta_{\left(2 \alpha T_{b}\right) \bmod 1},
\end{aligned}
$$

where $\tau \% T=\tau-\operatorname{round}(\tau / T) \cdot T$. More specifically, the asymptotic distributions of $\hat{R}_{x x *}(\alpha ; \tau)$ at some special points can be derived from (34). For instance, $\lim _{T \rightarrow \infty} \sqrt{T} \hat{R}_{x x *}\left(0 ; n T_{b}\right)$ follows asymptotic normal distributions with the parameters defined as

$$
\begin{aligned}
\Gamma_{x}\left(0 ; n T_{b}\right) & =S_{x x x^{*} x^{*}}^{0}(0)_{n T_{b}, n T_{b}}=A^{4} N_{c} T_{c} C_{x}\left(0 ; n T_{b}\right) \\
& =S_{x x x x}^{0}(0)_{n T_{b}, n T_{b}}=A^{4} N_{c} T_{c} e^{j 4 \pi f_{c} \tau} .
\end{aligned}
$$


It is well known that the DSSS signal has a low probability of intercept because of its low power spectrum density. The cooperative receiver can recover the information using the correlation operation or the matched filter. The advantage gained through the correlation receiver is called spreading gain, while the non-cooperative receiver can rarely benefit from it. One may notice that the PN sequence $c(n)$ does not appear in (35) and that the asymptotic covariance of $\hat{R}_{x x *}\left(0 ; n T_{b}\right)$ is proportional to spreading gain $N_{c}$, which means that non-cooperative receivers can also take advantage of the spectrum spreading gain using the CAF of the intercepted DSSS. In other words, the estimation of CAF resembles the correlation operation or the matched filter in some sense when the spreading code sequence $c(n)$ is unknown.

An example of the $\hat{R}_{x x *}(\alpha ; \tau)$ is plotted in Figure 2 . We notice that peaks appear in the regions $n T_{b}-T_{c}<\tau<$ $n T_{b}+T_{c}, n=1,2$ and $-1 / T_{b}<\alpha<1 / T_{b}$ just as discussed above.

\section{Applications and asymptotic performance analyses} From the properties of the CAF estimation mentioned in the above section, it can be seen that the CAF can be used to check the presence of a DSSS, and it also has the potential of recognizing a DSSS. The following assumptions are considered:

(A1) The intercepted DSSS signal $r(t)$ is BPSK-modulated both for the information and the PN code sequence as in (10) and (11). The pulse shape $q(t)$ is assumed to be rectangular without loss of generality.

(A2) The PN code sequence $c(n)$ is unknown.

(A3) The bit period $T_{b}$ is known. However, in practice, $T_{b}$ can also be estimated using the CAF of $r(t)$, while the estimation is not discussed here.

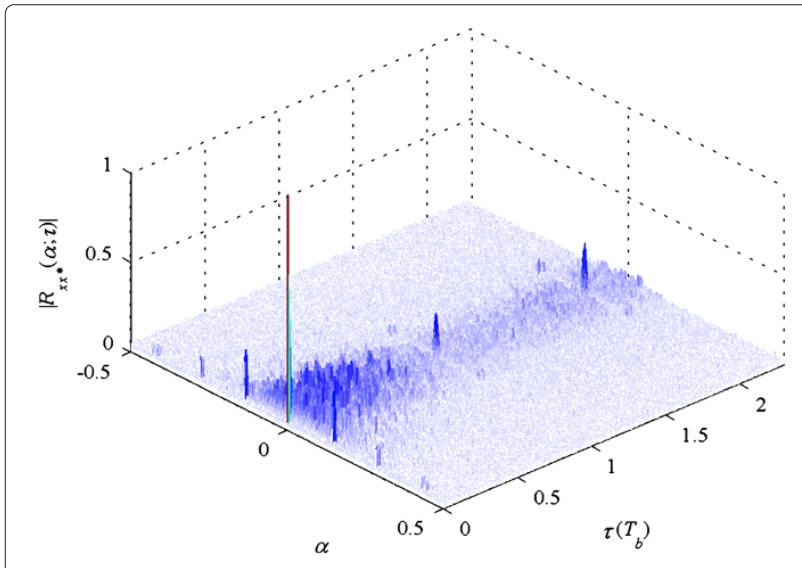

Figure 2 An example of the estimated CAF of $x$.
(A4) The signal $r(t)$ is oversampled. The sample period $T_{s}$ is a fraction of $T_{c}$, and the sample clock is not necessarily synchronized to the PN code in practice.

For both the detection and recognition, a unified binary hypothesis testing problem is formulated as follows:

$$
\begin{aligned}
& H_{0}: r(t)=y(t)+n(t) \\
& H_{1}: r(t)=x(t)+n(t)
\end{aligned}
$$

where $y(t)$ is a simple PAM signal defined as

$$
y(t)=A \sum_{k=-\infty}^{+\infty} a(k) q\left(t-k T_{c}\right) e^{j 2 \pi f_{c} t+\theta_{c}},
$$

and $x(t)$ is defined as in Equation 10. Equation 36 is a typical formulation of a classification problem, but when $y(t)=0$, Equation 36 turns into a special case as a detection problem.

From the discussion in the former section, it is seen that peaks appear in particular regions for the magnitude of the CAF of $r(t)$. We find that the hypothesis testing problem is actually equivalent to checking whether the covariance of the CAF at $\tau=n T_{b}, n \neq 0$, and $\alpha=0$ is over a threshold or not. Thus, we present the test statistic as follows:

$$
\lambda=\frac{T}{N_{0}^{2}} \sum_{n=1}^{N} \hat{R}_{r r *}\left(0 ; n T_{b}\right) \hat{R}_{r r *}^{*}\left(0 ; n T_{b}\right),
$$

where $N$ is the number of the lags. In (38), $\hat{R}_{r r *}\left(0 ; n T_{b}\right)$ represents the estimated CAF from the received DSSS $r(t)$

$$
\hat{R}_{r r^{*}}(\alpha ; \tau)=\frac{1}{T} \sum_{t=-T / 2}^{T / 2-1} r(t) r^{*}(t-\tau) e^{-j \alpha t} .
$$

For setting a threshold for the hypothesis testing problem, the asymptotic distribution of $\lambda$ will be derived next.

To get the asymptotic distribution of $\lambda$, it is necessary to get the asymptotic distribution of $\hat{R}_{r r *}\left(0 ; n T_{b}\right)$ first. The asymptotic distribution of $\hat{R}_{r r *}\left(0 ; n T_{b}\right)$ under $H_{i}, i=0,1$ is as follows:

$\lim _{T \rightarrow \infty} \sqrt{T} \hat{R}_{r r *}\left(0 ; n T_{b}\right) \stackrel{D}{=} C N\left(0, \Gamma_{r}^{i}\left(0 ; n T_{b}\right), C_{r}^{i}\left(0 ; n T_{b}\right)\right), n \neq 0$,

where $\Gamma_{r}^{i}\left(0 ; n T_{b}\right)$ and $C_{r}^{i}\left(0 ; n T_{b}\right)$ are the asymptotic covariance and relation under $H_{i}$ defined similar to (24) and (25) which can be expressed for $T$ that is large enough as follows (see Appendix 1 for more details):

$$
\begin{aligned}
& \Gamma_{r}^{0}\left(0 ; n T_{b}\right)=S_{r r r^{*} r^{*}}^{0}(0)_{n T_{b}, n T_{b}}=N_{0}^{2}+2 A^{2} N_{0}+A^{4} T_{c} \\
& C_{r}^{0}\left(0 ; n T_{b}\right)=S_{r r r r}^{0}(0)_{n T_{b}, n T_{b}}=A^{4} T_{c} e^{j 4 \pi f_{c} n T_{b}} \\
& \Gamma_{r}^{1}\left(0 ; n T_{b}\right)=S_{r r r^{*} r^{*}}^{0}(0)_{n T_{b}, n T_{b}}=N_{0}^{2}+2 A^{2} N_{0}+A^{4} N_{c} T_{c} \\
& C_{r}^{1}\left(0 ; n T_{b}\right)=S_{r r r r}^{0}(0)_{n T_{b}, n T_{b}}=A^{4} T_{b} e^{j 4 \pi f_{c} n T_{b}}
\end{aligned}
$$


The notation $N_{0}$ in (41) shows the power spectrum density of the complex additive white Gaussian noise $n(t)$ as in (9). From (41), it is easy to find the difference between the simple PAM signal and the DSSS signal. The greater the spectrum spreading gain $N_{c}$ is, the more different the test statistic $\lambda$ becomes.

Suppose that $\hat{R}_{r r *}\left(0 ; n T_{b}\right) \hat{R}_{r r *}^{*}\left(0 ; n T_{b}\right) n=1,2, \ldots, N$ is statistically independent and identically distributed. Therefore, based on the central limit theorem, when $N$ is considerably large, the distribution of $\lambda$ approaches Gaussian distribution, i.e.,

$$
\lim _{T \rightarrow \infty} \lambda \mid H_{i} \stackrel{D}{=} N\left(m_{i}, \sigma_{i}^{2}\right)
$$

with

$$
\begin{aligned}
m_{0} & =\frac{N}{N_{0}^{2}} E\left[\hat{R}_{r r *}\left(0 ; n T_{b}\right) \hat{R}_{r * *}^{*}\left(0 ; n T_{b}\right)\right] \\
& =\frac{N}{N_{0}^{2}}\left(N_{0}^{2}+2 A^{2} N_{0}+A^{4} T_{c}\right) \\
m_{1} & =\frac{N}{N_{0}^{2}}\left(N_{0}^{2}+2 A^{2} N_{0}+A^{4} N_{c} T_{c}\right)
\end{aligned}
$$

and

$$
\begin{aligned}
\sigma_{0}^{2}= & \frac{N T^{2}}{N_{0}^{4}} E\left\{\left[\hat{R}_{r r *}\left(0 ; n T_{b}\right) \hat{R}_{r r *}^{*}\left(0 ; n T_{b}\right)\right.\right. \\
& \left.\left.-E\left[\hat{R}_{r r *}\left(0 ; n T_{b}\right) \hat{R}_{r r *}^{*}\left(0 ; n T_{b}\right)\right]\right]^{2}\right\} \\
= & \frac{N}{N_{0}^{4}}\left[\left(N_{0}^{2}+2 A^{2} N_{0}+A^{4} T_{c}\right)^{2}+\left(A^{4} T_{c}\right)^{2}\right] \\
\sigma_{1}^{2}= & \frac{N}{N_{0}^{4}}\left[\left(N_{0}^{2}+2 A^{2} N_{0}+A^{4} N_{c} T_{c}\right)^{2}+\left(A^{4} N_{c} T_{c}\right)^{2}\right],
\end{aligned}
$$

where the derivation of the variants uses the fourth moment of $\hat{R}_{r r *}\left(0 ; n T_{b}\right)$ (see Appendix 2 for more details). Particularly, when $y(t)=0$, Equation 36 is reduced to a simple detection problem, which can be used to decide whether a DSSS signal exists in the intercepted signal or not. In this case, the parameters of (42) can be reduced to a simple detection version as

$$
m_{0}=N
$$

and

$$
\sigma_{0}^{2}=N \text {. }
$$

By following the conclusion of (42), we present a DSSS detector based on a constant false alarm rate approach. A threshold $\eta$ should be worked out according to a probability of false alarm which is defined as $P_{F} \triangleq \operatorname{Pr}\left\{\lambda>\eta \mid H_{0}\right\}$. Because of the distribution of $\lambda$ as in (42), $\eta$ can be found by searching for the Gaussian distribution tables, such that $P_{F}=\operatorname{Pr}\{\lambda \geq \eta\}$. Then, the detector is given as

$$
\lambda \underset{H_{0}}{\stackrel{H_{1}}{\geq}} \eta,
$$

which means that $H_{1}$ holds if $\lambda \geq \eta$, and vice versa. Since the threshold $\eta$ has been set, the probability of detection can be evaluated using the distribution of $\lambda$ under $H_{0}$, which is defined as $P_{D} \triangleq \operatorname{Pr}\left\{\lambda \geq \eta \mid H_{1}\right\}$.

Therefore, the detection algorithm for the presence of the DSSS is implemented using the following steps:

Step 1. Using (39), estimate the CAF of received signal $r(t)$.

Step 2. Calculate the test statistic $\lambda$ as in (38).

Step 3. Set an expected value of false alarm rate $P_{F}$. Using $P_{F}$, find a threshold $\eta$ by checking the Gaussian distribution tables, such that $P_{F}=\operatorname{Pr}\{\lambda \geq \eta\}$

Step 4. If $\lambda \geq \eta$, declare that $H_{1}$ holds, which means that a DSSS is present in the received signal or the type of the signal is DSSS, and vice versa.

\section{Simulations}

To verify the accuracy of asymptotic Gaussian distributions for the test statistics of the recognition problem where $y(t)$ is given by (37), histograms of estimated $\lambda$ for simulated signals are plotted in Figure 3, where they are compared with the corresponding theoretical values. The DSSS signals are generated using (10) with $N_{c}=31$, $A=1, N=100$ and for the SNR being fixed at $-10 \mathrm{~dB}$, where the SNR is defined, in decibels, as

$$
\mathrm{SNR}=10 \log _{10} \frac{A^{2}}{N_{0}} .
$$

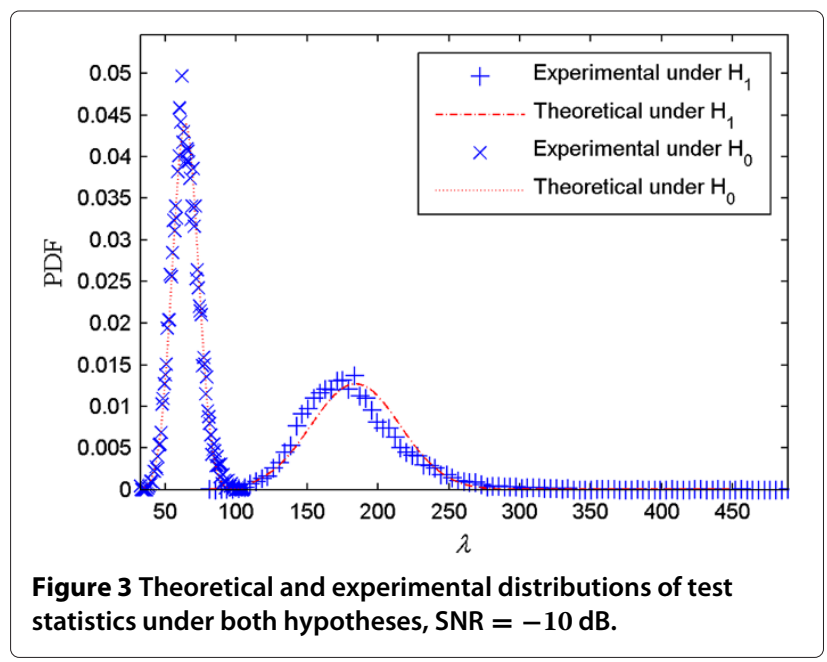




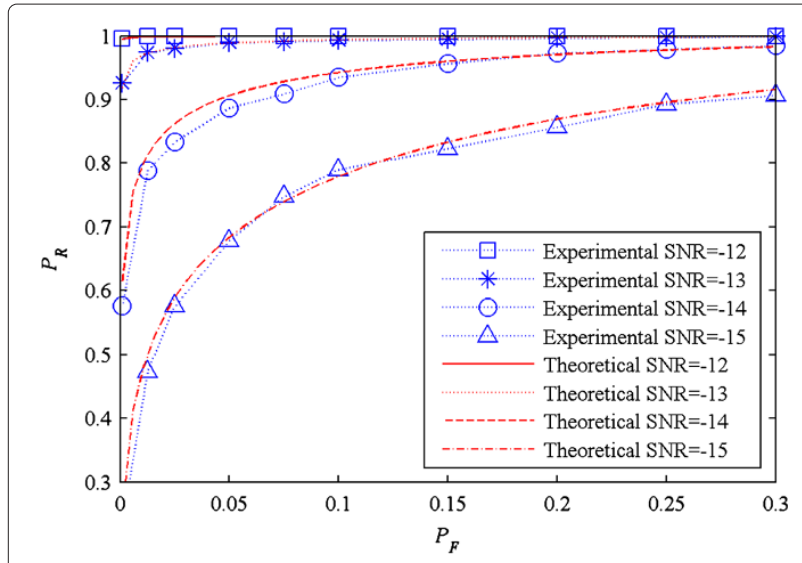

Figure $4 P_{D}$ vs. $P_{F}$ in the recognition problem.

The chip period $T_{c}=8 T_{s}$ and the carrier frequency $f_{c}=0.022 / T_{s}$, where $T_{s}$ is the sample period. Set the observation interval $T$ be $10^{4} T_{s}$. The theoretical distributions of $\lambda$ getting through asymptotic analysis are represented by lines, and the experimental ones getting through simulations are represented by markers.

The detectability of the presented test statistics is investigated in terms of the receiver operation characteristic. The probabilities of recognition $P_{R}$ are plotted versus the probability of false alarm $P_{F}$ for the SNRs from -12 to $-15 \mathrm{~dB}$ in Figure 4 where the theoretical curves are represented by lines, while the experimental results are represented by markers. The lowest false alarm probability in the simulation in Figure 4 is $10^{-3}$, so from the experiments, one can see that the recognition probability is above 0.9 when $P_{F}=10^{-3}$ and the $\mathrm{SNR}=-13 \mathrm{~dB}$.

When the PAM signal $y(t)=0$ in the hypothesis testing problem of Equation 36, the problem becomes a detection problem. The probabilities of detection $P_{R}$ versus the probability of false alarm $P_{F}$ are plotted in Figure 5 for -

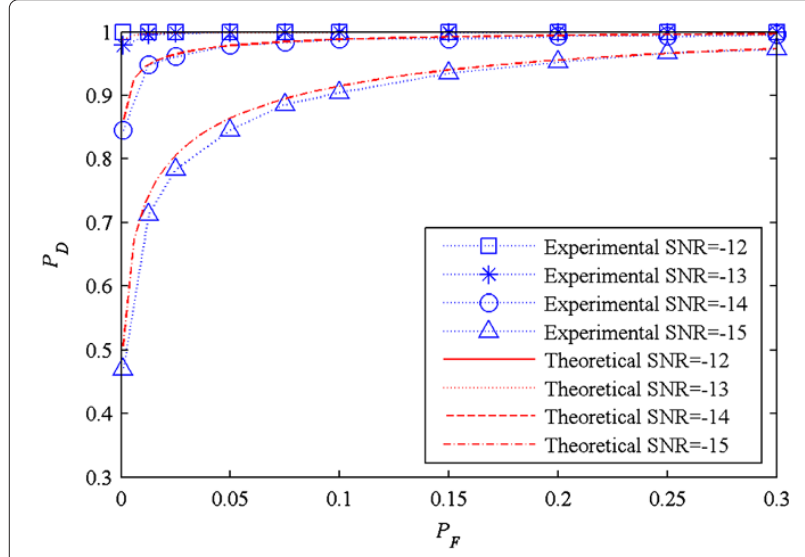

Figure $5 P_{D}$ vs. $P_{F}$ in the detection problem.

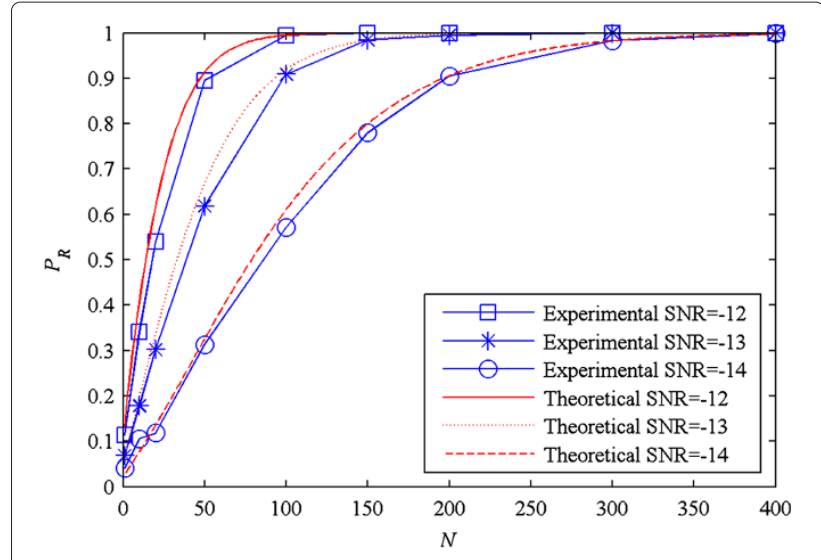

Figure $6 P_{D}$ vs. $N$ when $P_{F}=0.001$

the SNRs from -12 to $-15 \mathrm{~dB}$. The condition in Figure 5 is the same with that in Figure 4, while the only discrimination is the absence of $y(t)$ in Figure 5. As we can see from the comparison of Figures 4 and 5, the detection probabilities are slightly greater than the recognition probabilities for the absence of $y(t)$ in the detection problem.

Numerical experiments are carried out to evaluate the influence of the summation number of the lags $N$ on the performance of the DSSS recognition. The results plotted in Figure 6 demonstrate that the probability of recognition $P_{R}$ increases rapidly as $N$ becomes larger, and it becomes almost 1 at a moderate number of $N$ for which the computational load will not be dramatically unacceptable. When SNR becomes lower, the situation becomes worse in which increasing $N$ will not make the $P_{R}$ increase rapidly. Thus, it is necessary for one to choose a moderate $N$ to achieve an optimal tradeoff between the performance and the computational load. The computational load of the algorithm is the order of $\left[N T\left(1+\log _{2} T\right)\right]$ when the FFT is used.

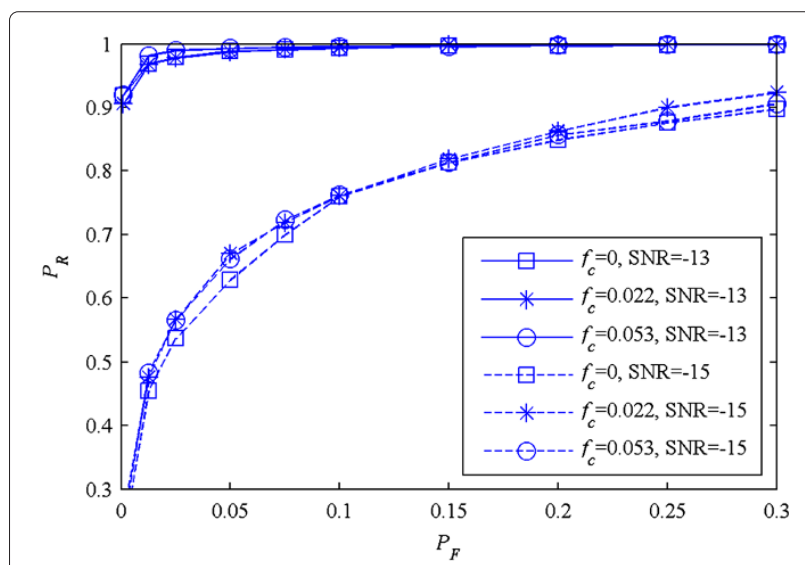

Figure $7 P_{D}$ vs. $P_{F}$ with carrier offsets. 


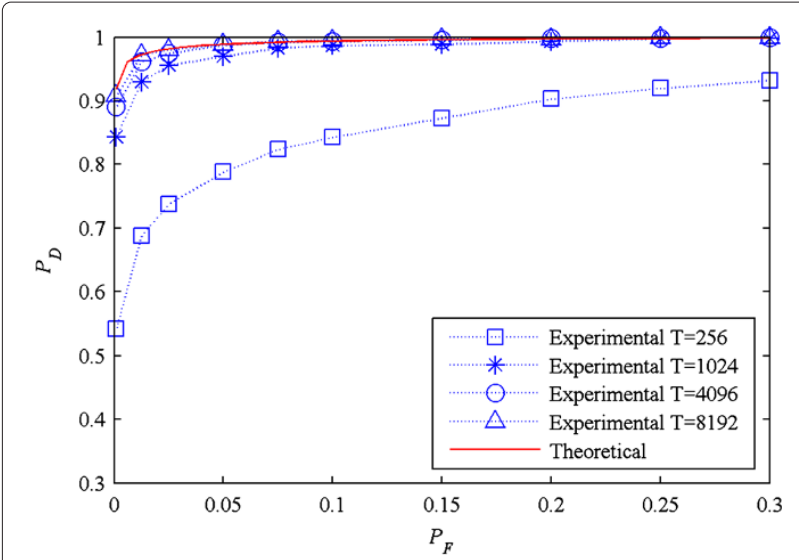

Figure $8 P_{D}$ vs. $P_{F}$ with different $T$.

A simulation is also performed to evaluate the effect of the carrier frequency $f_{c}$ on the recognition problem of DSSS. In Figure 7, the square, asterisk, and circle markers represent $P_{R}$ when $f_{c}=0,0.022$ and $0.053 / T_{s}$, respectively. The simulation results in Figure 7 show that $f_{c}$ cannot affect $P_{R}$, which verify the theoretical analysis of (42).

Next, the simulation is concerned with the asymptotic performance of the proposed test statistics. Figure 8 shows experimental probabilities of recognition when $T=256$, $1,024,4,096$, and 8,192 with respect to theoretical values. Note that when the observation interval $T$ increases, the experimental probabilities rapidly approach the theoretical ones. The simulation demonstrates that it does not need a very large $T$ to get a good performance in applications.

\section{Conclusion}

The asymptotic distributions of the estimated CA of DSSS signals are derived. The variance of the estimation resembles a series of thumbtack forms, and the cyclic period equals the symbol period. Though it vanishes as the observation time becomes infinite, the properties of the estimated CA can be used in the detection and recognition problem of DSSS signals. A good agreement is obtained between theoretical and simulation results. The simulations also show that non-cooperative receivers can also take advantage of the spectrum spreading gain using the CAF of the intercepted DSSS just as the cooperative receivers do.

\section{Appendix 1}

Using the superimposition of the two independent zeromean complex time series $(r(t)=x(t)+n(t))$, the cyclic cross spectrum can be expressed as [8]

$$
\begin{aligned}
S_{r r r r}^{\gamma}(\omega)_{\tau, \rho}= & S_{n n n n}^{\gamma}(\omega)_{\tau, \rho}+S_{x x x x}^{\gamma}(\omega)_{\tau, \rho}+S_{x n x n}^{\gamma}(\omega)_{\tau, \rho} \\
& +S_{n x n x}^{\gamma}(\omega)_{\tau, \rho}+S_{x n n x}^{\gamma}(\omega)_{\tau, \rho}+S_{n x x n}^{\gamma}(\omega)_{\tau, \rho} .
\end{aligned}
$$

Each quantity in (49) can be evaluated and expressed as

$$
\begin{aligned}
S_{n n n n}^{\gamma}(\omega)_{\tau, \rho} & =S_{x n x n}^{\gamma}(\omega)_{\tau, \rho}=S_{n x n x}^{\gamma}(\omega)_{\tau, \rho}=S_{x n n x}^{\gamma}(\omega)_{\tau, \rho} \\
& =S_{n x x n}^{\gamma}(\omega)_{\tau, \rho}=0
\end{aligned}
$$

because $n(t)$ is a complex additive white Gaussian noise. The conjugate cyclic cross spectrum can be expressed as

$$
\begin{gathered}
S_{r r r^{*} r^{*}}^{\gamma}(\omega)_{\tau, \rho}=S_{n n n^{*} n^{*}}^{\gamma}(\omega)_{\tau, \rho}+S_{x x x^{*} x^{*}}^{\gamma}(\omega)_{\tau, \rho}+S_{x n x^{*} n^{*}}^{\gamma}(\omega)_{\tau, \rho} \\
\quad+S_{n x n^{*} x^{*}}^{\gamma}(\omega)_{\tau, \rho}+S_{x n n^{*} x^{*}}^{\gamma}(\omega)_{\tau, \rho}+S_{n x x^{*} n^{*}}^{\gamma}(\omega)_{\tau, \rho}, \quad \text { (51) }
\end{gathered}
$$

where each quantity can be evaluated and expressed as

$$
\begin{aligned}
& S_{n n n^{*} n^{*}}^{\gamma}(\omega)_{\tau, \rho}=N_{0}^{2} \eta_{\gamma}\left(\delta_{\tau-\rho}+\delta_{\tau+\rho} e^{-j \tau \omega}\right) \\
& S_{x n x^{*} n^{*}}^{\gamma}(\omega)_{\tau, \rho}=N_{0} R_{x x *}(\gamma ; \tau-\rho) e^{-j \omega(\tau-\rho)} \\
& S_{n x n^{*} x^{*}}^{\gamma}(\omega)_{\tau, \rho}=N_{0} R_{x x *}(\gamma ; \rho-\tau) e^{-j \gamma \tau} \\
& S_{x n n^{*} x^{*}}^{\gamma}(\omega)_{\tau, \rho}=N_{0} R_{x x *}(\gamma ; \rho+\tau) e^{-j \omega \tau} \\
& S_{n x x^{*} n^{*}}^{\gamma}(\omega)_{\tau, \rho}=N_{0} R_{x x *}(\gamma ;-\rho-\tau) e^{j \omega \rho} e^{j \gamma \tau} .
\end{aligned}
$$

By using (32), substituting (52) into (51) and (50) into (49), the asymptotic covariance and relation under $H_{i}, i=0,1$ can be respectively expressed as in (41).

\section{Appendix 2}

The characteristic function of a zero-mean complex Gaussian variable $z \stackrel{D}{=} C N(0, \Gamma, C)$ is given by [14]

$$
\Phi_{z}(\omega)=\exp \left\{-\frac{1}{4}\left[\omega^{*} \Gamma \omega+\operatorname{Re}\left(\omega^{* 2} C\right)\right]\right\} .
$$

The relation between the $m$ th moment of a complex random variable and the characteristic function is given in [15]. The fourth-order moment of $z$ can be achieved by

$$
\begin{aligned}
E\left[z^{2} z^{* 2}\right] & =\left.16 \frac{\partial^{4} \Phi_{z}(\omega)}{\partial \omega^{2} \partial \omega^{* 2}}\right|_{\omega=0, \omega^{*}=0} \\
& =2 \Gamma^{2}+|C|^{2} .
\end{aligned}
$$

\section{Competing interests}

The authors declare that they have no competing interests.

\section{Author details}

${ }^{1}$ Department of Electronic Engineering, Xidian University, Xi'an 710071, China. ${ }^{2}$ Shandong Institute of Aerospace Electronic Technology, Yantai 264670, China.

\section{Acknowledgements}

We want to thank the helpful comments and suggestions from the anonymous reviewers. This research was supported partially by the Fundamental Research Funds for the Central Universities (grant no. K5051202002).

Received: 1 February 2013 Accepted: 19 May 2013

Published: 28 May 2013 


\section{References}

1. G Burel, in IEEE Int. Symp. on Intelligent Signal Processing and Communications Systems (ISPAC'2000). Detection of spread spectrum transmission using fluctuations of correlation estimators (IEEE Honolulu, 5-8 Nov 2000)

2. G Burel, C Bouder, O Berder, in IEEE Global Telecommunications Conference GLOBECOM'01, San Antonio, TX. Detection of direct sequence spread spectrum transmissions without prior knowledge (IEEE New York, 2001), pp. 236-239

3. Z Dong, $\mathrm{H} \mathrm{Hu}, \mathrm{H} \mathrm{Yu}$, The detection, symbol period and chip width estimation of DSSS signals based on delay-multiply, correlation and spectrum analysis. J. Electron. Inf. Technol. 30, 840-842 (2008)

4. Z Deng, L Shen, N Bao, B Su, J Lin, D Wang, in 2011 International Conference on Wireless Communications and Signal Processing (WCSP). Autocorrelation based detection of DSSS signal for cognitive radio system (IEEE New York, 2011), pp. 608-612

5. H Yang, X Wang, Y Zou, L Wang, Novel approach to detect the spread spectrum signal and estimate period of PN based on blind source separation. J. Comput. Inf. Syst. 7, 637-644 (2011)

6. U Ahnström, J Falk, P Händel, M Wikström, in Nordic Matlab Conference. Detection and direction-finding of spread spectrum signals using correlation and narrowband interference rejection (COMSOL A/S Copenhagen, 21-22 Oct 2003)

7. Dandawaté A V, GB Giannakis, Statistical tests for presence of cyclostationarity. IEEE Trans. Signal Proc. 42, 2355-2369 (1994)

8. P Rostaing, E Thierry, T Pitarque, Asymptotic performance analysis of cyclic detectors. IEEE Trans. Commun. 47, 10-13 (1999)

9. M Tsatsanis, G Giannakis, Blind estimation of direct sequence spread spectrum signals in multipath. IEEE Trans. Signal Proc. 45, 1241-1252 (1997)

10. G Burel, E Bouder, in Proc. Int. Symp. Military Commun. (MILCOM2000), Los Angeles. Blind estimation of the pseudo-random sequence of a direct sequence spread spectrum signals (IEEE New York, 2000), pp. 967-970

11. A Napolitano, M Tanda, Blind parameter estimation in multiple-access systems. IEEE Trans. Commun. 49, 688-698 (2001)

12. WA Gardner, A Napolitano, L Paura, Cyclostationarity: Half a century of research. Signal Process. 86, 639-697 (2006)

13. A Napolitano, Cyclic higher-order statistics: input/output relations for discrete- and continuous-time MIMO linear almost-periodically time-variant systems. Signal Process. 42, 147-166 (1995)

14. B Picinbono, Second-order complex random vectors and normal distributions. IEEE Trans. Signal Process. 44, 2637-2640 (1996)

15. J Eriksson, E Ollila, V Koivunen, Essential statistics and tools for complex random variables. IEEE Trans. Signal Proc. 58, 5400-5408 (2010)

\section{Submit your manuscript to a SpringerOpen ${ }^{\circ}$ journal and benefit from:}

- Convenient online submission

- Rigorous peer review

- Immediate publication on acceptance

- Open access: articles freely available online

- High visibility within the field

- Retaining the copyright to your article

Submit your next manuscript at $\boldsymbol{\nabla}$ springeropen.com 